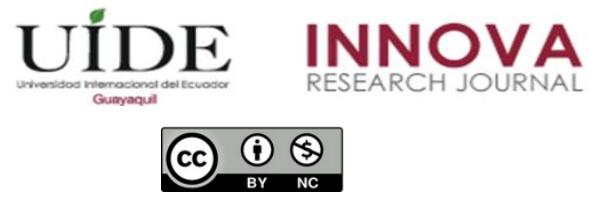

INNOVA Research Journal, ISSN 2477-9024

(Enero - Abril 2021). Vol. 6, No.1 pp. 129-144

DOI: https://doi.org/10.33890/innova.v6.n1.2021.1454

URL: http://revistas.uide.edu.ec/index.php/innova/index

Correo: innova@uide.edu.ec

\title{
Diferencias salariales y segregación ocupacional en el mercado laboral del Ecuador
}

\section{Salary differences and occupational segregation in the Ecuadorian labor market}

Patricia Alexandra Uriguen Aguirre

(D) https://orcid.org/0000-0003-3095-8765

Josue Daniel Martínez Valarezo

https://orcid.org/0000-0003-4372-5290

Vinicio Enrique Carrión Ayala

(D) https://orcid.org/0000-0003-0699-0722

Universidad Técnica de Machala, Ecuador

Autor para correspondencia: puriguen@utmachala.edu.ec; jdmartinez_est@utmachala.edu.ec; vcarrion2@utmachala.edu.ec

Fecha de recepción: 14 de junio de 2020 - Fecha de aceptación: 16 de octubre de 2020

\section{Resumen}

El propósito de este estudio es analizar la relación entre las diferencias salariales entre hombres y mujeres y la segregación ocupacional de género afecta a la misma, utilizando datos de clasificación ocupacional. Se examinan si existe una relación estadísticamente significativa entre el empleo y el tamaño de la parte inexplicable de la brecha salarial de género. La metodología se fundamenta con estimaciones que identifica las determinantes del ingreso laboral con el método de descomposición Oaxaca-Blinder (1973), mismo que permite examinar las diferencias en las brechas salariales de genero entre grupos ocupacionales, el análisis se basa en datos individuales de diez años (20102019) disponibles en Ecuador. Los resultados mencionan que no existe relación fuerte entre la segregación ocupacional y el tamaño de las diferencias inexplicables en los salarios. Las variables que explican este contraste son la edad, tipo título, título profesional, y estado civil. Las recomendaciones se basan en robustecer la participación y permanecía de las mujeres en el mercado laboral de igual forma mediante el cumplimiento de leyes y normativas existentes.

Palabras claves: brecha salarial; segregación ocupacional; discriminación salarial; género.

\begin{abstract}
The purpose of this study is to analyze the relationship between the wage differences between men and women and the occupational gender segregation that affects it, using data from occupational classification. It is examined whether there is a statistically significant relationship between employment and the size of the unexplained part of the gender pay gap. The methodology is based on criteria that identify the determinants of labor income with the Oaxaca-Blinder decomposition method (1973), which allows examining the differences in gender wage gaps between occupational
\end{abstract}


groups. The analysis is based on individual data from ten years (2010-2019) available in Ecuador. The results mention that there is no strong relationship between occupational segregation and the size of the inexplicable differences in wages. The variables that explain this contrast are age, title type, professional title and marital status. The recommendations are based on strengthening the participation and permanence of women in the labor market in the same way by complying with current laws and regulations.

Keywords: wage gap; occupational segregation; wage discrimination; gender.

\section{Introducción}

Distintas investigaciones manifiestan que las diferencias de género en los salarios son una particularidad sólida en mercados laborales contemporáneos en la mayoría de las economías desarrolladas (Banerjee, 2014; Brynin y Perales, 2016). Considerando que solo una fracción de la brecha salarial entre hombres y mujeres puede explicarse por las diferencias en su productividad. Investigaciones en varios países indica que existe una relación entre la segregación ocupacional y el nivel de los salarios, pero la forma de esta relación no se encuentra bien establecida. Ciertas investigaciones mencionan que la relación es lineal: las labores femeninas pagan menos que las labores masculinas (Kunze, 2008; Sławińska, 2020). Algunos países han estudiado la relación entre la segregación ocupacional y el tamaño de la brecha salarial de género, en ciertos casos las conclusiones han sido no concluyentes. Blau \& Kahn (2003) encontrando evidencia débil entre la brecha salarial de género y la segregación ocupacional correlacionadas de forma positiva.

En investigación realizada por Hakim (1998) encontró una relación no lineal entre la brecha salarial de género es relativamente baja en ocupaciones mixtas, por lo que existe un considerable desacuerdo sobre si esta brecha en realidad refleja la discriminación de género en el mercado laboral o más bien las diferencias de productividad entre los trabajadores. La mayor parte de estudios para medir la segregación ocupacional de los trabajadores utilizan datos agregados en la regresión de la brecha salarial de género. La segregación ocupacional es una característica en los mercados laborales y se considera que tiene un impacto salarial pronunciado (Anker, 1997). Las ocupaciones donde predominan las mujeres generalmente pagan menos que aquellas donde predominan los hombres (England, Herbert, Kilbourne, y L, 1994; Blau y Kahn, 2003). Amplios estudios de investigación han tratado de encontrar las causas y con frecuencia mencionan teorías importantes, como la teoría del capital humano Tam (1997) y la teoría del rol de género (Ochsenfeld, 2014). La evidencia reciente muestra que tanto la segregación ocupacional como la diferencia salarial están disminuyendo lánguidamente (Brynin y Perales, 2016).

El estudio profundiza la literatura existente al centrarse en el análisis de ocupaciones detalladas. Se estima la brecha salarial de género para cada uno de los grupos ocupacionales y se evalúan en qué medida las diferencias están justificadas por las características de los trabajadores. Una vez hecho esto, el documento examina si existe una relación estadísticamente significativa entre la proporción de hombres y mujeres en la ocupación y el tamaño de la parte inexplicable de la brecha salarial. La parte inexplicable de la brecha salarial generalmente se trata en la literatura como una estimación de la discriminación salarial (Jurajda, 2003; Goraus, Tyrowicz, y Van der Velde, 2017). Sin embargo, como es bien sabido, los efectos de 
discriminación pueden exagerarse si faltan algunas variables explicativas importantes. Por el contrario, la parte inexplicable también subestimará la discriminación si algunas de las variables explicativas han sido influenciadas por la discriminación (Barón y Cobb-Clark, 2010).

La (Constitucion de la Republica del Ecuador, 2008, última modificación 2015) en su artículo 66, numeral 4 menciona la igualdad formal, igualdad material y no discriminación, incluyendo el principio que configura la igualdad de género para las personas, y en el artículo 8 inciso (a) de la (Ley orgánica integral para prevenir y erradicar la violencia contra las mujeres, 2018) contempla los principios rectores de igualdad y no discriminación para el género en todo el territorio nacional.

En Ecuador de acuerdo a García y Cortez (2011) la fuerza femenina en el mercado laboral no es uniforme y depende de distintos factores como edad, educación y características familiares, así mismo existe el hecho de que las mujeres disminuyen su participación cuando en su seno familiar existe un familiar con ingresos diferentes a los que ella aporta. López y Sarmiento (2019) menciona que la educación y el mercado laboral están ligados por lo que cuando se posee conocimiento de otro idioma se desarrolla ampliamente la actividad laboral.

Aunque la actividad laboral de las mujeres es cada vez mayor, su participación se concentra en el sector terciario o de servicios y la diversificación es escasa para los diferentes tipos de ocupación (Albuja-Echeverría y Enríquez-Rodríguez, 2018). Existiendo la discriminación salarial no percibida por la segregación ocupacional, debido a que las mujeres no mantienen iguales puestos que los hombres, desempeñándose en empleos menos valorizados con ingresos menores, considerándose a los hombres con mejores capacidades y aptitudes preponderando la discriminación. Nace así la motivación de la investigación en la división y explicación de la desigualdad de género de los diferentes principios que se encuentran en el mercado laboral ecuatoriano, provocando un fenómeno cultural y social por su género, salarios distintos de hombres y mujeres en la población ecuatoriana. La división de las diferentes causas podrán explicar, manifestar e ilustrar lo abstracto e injusto de la brecha salarial en el Ecuador, es por eso que se considera la importancia de este estudio.

En consecuencia, el objetivo de este trabajo es agregar a la literatura empírica un análisis de la desigualdad de género y su implicancia en el tamaño de las diferencias salariales en el mercado laboral ecuatoriano.

\section{Revisión de Literatura}

Las brechas salariales pueden varían sustancialmente entre países (Blau y Kahn, 2003). pueden cambiar dependiendo del país basándose mucho en lo que son los niveles de experiencia y la cantidad de ingresos que tiene el país, debido a esto se puede observar que la brecha salarial entre hombres y mujeres existe dependiendo del país, con mayor o menor afectación, causales como educación, edad, salud, experiencia laboral, acceso a la tecnología (Ospina, 2015; Novillo, Sarmiento, Ollague, y Ramón, 2017; Uriguen, Vega, y Luna-Romero, 2020), provocan la brecha salarial por el cúmulo de características en el capital humano se convierte en una idoneidad al momento de realizar las funciones dentro de los mercados laborales. La ruptura de un equilibrio 
que normativamente debería existir tanto entre hombres y mujeres basándose en una igualdad de género Bergmann (1986), se presentan por las barreras del hacinamiento. Considerándose diferencias en las actividades laborales femeninas y masculinas England, Herbert, Kilbourne, y L (1994), por lo que los salarios más bajos son producidos por actividades femeninas.

La literatura empírica no es concluyente, diversas investigaciones confirman que la brecha salarial de género es más alta en ocupaciones que requieren niveles de educación superior Blau y Kahn, 2003; Evertsson, y otros (2009), encontrando una relación positiva débil. Estudio realizado en Australia de los sectores públicos y privados de la brecha salarial y la segregación ocupacional por género de Barón y Cobb-Clark (2010), encontraron que la brecha es plana en el sector público y tiene aumentos dependiendo de los salarios para el sector privado. Efectos similares fueron encontrados para países como Gran Bretaña, Suiza y Alemania en investigación por datos de panel realizada por Murphy y Oesch (2015), en la que son mayores las sanciones en el sector privado que en el público.

Investigaciones realizadas en diferentes contextos han descubierto diferencias salariales y segregaciones ocupacionales, Alcañiz y Monteiro (2016), encontraron que, en España y Portugal existe una mayor segregación que en Italia, esto se debe el mayor número de féminas participando en la actividad laboral. En España Milan Vásquez de la Torre, Santos, y Perez (2015), descubrieron que existe una alta segregación por sexo en determinadas profesiones, son las mujeres las que ocupan las categorías con poca calificación, remuneración y prestigio social.

De acuerdo con lo mencionado por Aguilar y Vera (2016), aunque si bien, la remuneración no depende únicamente del conocimiento adquirido por las personas, aquí también se hace evidente la segregación salarial, debido a que, a pesar de que las mujeres se encuentren preparadas y estudiadas, sin importar su nivel, recibirán una remuneración menor frente a los hombres. Caicedo (2015) ponía de ejemplo lo que ocurre en Estados Unidos, por motivo de ocupación y fuerza humana, donde los hombres alcanzan un salario mayor que las mujeres, debido a que no solo se encuentran ocupando rangos más altos, sino que también se estima que en su mayoría se ocupan del cargamento pesado. Mientras que, para los países latinoamericanos, se estima que los hombres tienen un salario mayor, debido a que su trabajo se asocia a mayor eficiencia, conocimiento y capacidad de esfuerzo (Bustos, 2008).

En cuanto a lo que ocurre específicamente en el Ecuador, Jumbo y Granda (2014) estiman que uno de los factores considerados para asignar la remuneración a los colaboradores, sin importar su género, es un manejo adecuado del conocimiento acorde con el puesto en el que la persona aspira a laborar. Para Zuñiga (2006) no es válida esta afirmación, debido a que considera que la mujer percibe menos ingresos, afirmación que sustenta con datos estadísticos tomados desde el año 1990. Estos datos reflejan que las mujeres se encuentran en puestos altos en un $26 \%$, mientras que los hombres se desempeñan en mejores cargos y mayor remuneración en un $74 \%$.

\section{Mecanismos principales de diferencias salariales y segregación ocupacional}

Diversos estudios mencionan que coexiste una relación inversa entre la segregación ocupacional y los niveles de salarios: fuerza ocupacional de dominio femenino tienen salarios 
más bajos que los trabajadores en fuerza ocupacional dominadas por hombres. Esta investigación explica las diferencias salariales y la segregación ocupacional en el mercado laboral, basados en al menos uno de los tres mecanismos principales como son la teoría del capital Humano, teoría del hacinamiento, y sesgo de género (Simón, Ramos, y Sanromá, 2008; Oyinlade y Losen, 2014).

De acuerdo a la teoría del capital humano, donde se puede mencionar que existe una desigualdad profunda en el ámbito productivo, de igual manera en la remuneración que reciben hombres y mujeres (Mincer y Polachek, 1974; Garrido, 2007; Angulo, Quejada, y Yánez, 2012). Las mujeres cuentan con una realidad en la esfera doméstica por ser las principales cuidadoras de los niños, por lo tanto, tienen menor inversión de capital humano que los hombres. Por la misma razón los empleadores tienen preferencias con el capital humano masculino. De forma parecida la existencia de la brecha salarial de género expone la inclinación de las mujeres en optar por alternativas con horarios flexibles o valores salariales menores, con la finalidad de equilibrar el trabajo o con el rol de la vida familiar. Derivando a que no solo la segregación ocupacional, incluida el apego de lo tradicional de los roles de la vida familiar, logra lesionar a las mujeres en el ámbito laboral (Juárez y Aboites, 2008 Lavassani y Mohavedi, 2014).

Según la teoría del hacinamiento, en la que explica las diferencias de los salarios en el género por las barreras de ingreso de las mujeres en algunas actividades del mercado laboral (Bergmann, 1986). Siendo la oferta y la demanda del mercado que fijaran los salarios del mercado laboral. Considerándose algunas restricciones en ocupaciones con dominio femenino que es alta, provocando la disminución del promedio de los salarios. Con esta teoría las mujeres y los hombres se mantienen por grupos no competitivos, por lo que representa que se mantienen límites en los segmentos laborales, evitando reasignar hombres o mujeres de acuerdo a la actividad.

La tercera teoría se basa en el sesgo de género, siendo la actividad laboral femenina y masculina evaluada y pagada de manera diferentes por los empleadores England, Herbert, Kilbourne, y L (1994), prevaleciendo estas diferencias en el mercado laboral ecuatoriano.

\section{Materiales y Métodos}

Enfoque estándar documental bibliográfico de la literatura, análisis conceptual y teórico, con material bibliográfico y documental como: artículos científicos de alto impacto, bases de datos del Banco Central del Ecuador (BCE), indicadores labores de encuestas nacionales del Instituto Nacional de Estadísticas y Censos (INEC), encuesta nacional de empleo, desempleo y subempleo (ENEMDU), datos oficiales del Banco Mundial (BM).

Estimaciones extendidas con un modelo econométrico, explicando supuestos y variables de la desegregación salarial, con un nivel exploratorio de acuerdo a la composición del mercado laboral, actividades relacionadas al campo de estudio, mecanismo de paga, luego con un nivel descriptivo mediante el análisis de datos para identificar las características de la realidad del mercado laboral ecuatoriano, y finalmente un nivel explicativo conociendo y descubriendo causales existentes de hechos de la delimitación (Weichselbaumer y Winter-Ebner, 2005). 
De acuerdo a los métodos se aprecia el lógico general, mediante un análisis que explica de forma concreta y detallada los ingresos en el mercado laboral, con el deductivo en que la teoría económica expone la relación de las variables de estudio y de esta manera obtener las debidas conclusiones, bajo el método histórico recopilando información verdadera a lo largo del tiempo relacionada con las diferencias salariales y segregación ocupacional. Adicionalmente con el objetivismo en el que se presenta la realidad de cómo es percibido la investigación de acuerdo a los hechos y el subjetivismo validando el conocimiento con los resultados, aportes y conclusiones de los investigadores. Se aplicó la macroeconomía, ya que se estudió algunos sectores de forma grupal, empleando datos de magnitudes globales y para recopilar y juntar toda la información se utilizó el método de síntesis que permitió resumir y concretar la información. Por último, se manejó métodos auxiliares como el estadístico, que permitió la recolección, presentación, síntesis y análisis de los datos de la investigación, y el econométrico que permitió estudiar una variable en función de otras como es la discriminación en la diferencia salarial del Ecuador.

Para responder a la pregunta en qué medida la brecha salarial de género puede explicarse por las diferentes características de hombres y mujeres, se utiliza la descomposición de (Oaxaca, 1973; Blinder, 1973), la misma que descompone la diferencia salarial media en forma logarítmica, de acuerdo a un modelo de regresión lineal. Luego, se intenta responder a la pregunta de si existe una relación estadísticamente significativa entre la parte inexplicada de la brecha salarial de género y la proporción de hombres en el grupo ocupacional. Para verificar la solidez de los resultados, se utilizan dos tipos de descomposición ponderada.

\section{Datos y estrategia empírica descomposición Oaxaca-Blinder}

Datos de salarios y características individuales de empleados utilizados en este documento se obtuvieron de la base de datos de los reportes de mercado laboral de la dirección nacional de síntesis macroeconómica de subgerencia de programación y regulación del Banco Central del Ecuador (BCE), y del Instituto nacional de estadísticas y censos (INEC).

Los datos utilizados involucran subconjuntos de registros base, del último informe del Laboratorio de Dinámica Laboral y Empresarial, y que contribuyen al diseño e implementación de políticas públicas y a obtener un mayor conocimiento sobre la realidad productiva y laboral del país.

Considerando dos grupos, $A$ y $B$; la variable dependiente $Y$; y un conjunto de predictores (variables independientes). Siendo A: grupo de hombres y B: grupo de mujeres, la Y: logaritmo natural del ingreso, y los predictores son la educación y experiencia laboral. Por lo que se plantea la diferencia salarial media D

$$
\mathrm{D}=\mathrm{E}\left(\mathrm{Y}_{\mathrm{A}}\right)-\mathrm{E}\left(\mathrm{Y}_{\mathrm{B}}\right)
$$

Donde $\mathrm{E}(\mathrm{Y})$ denota el valor esperado de la variable dependiente, parte que se explica con los predictores de cada grupo, la base se centra en el modelo lineal.

$$
\mathrm{Y}_{l}=\mathrm{X}_{l}^{\prime} \beta_{l}+\varepsilon_{l}
$$


Se considera a $l$ como A o B, X vector que involucra a predictores y a una constante, $\beta$ es vector de parámetros y $\varepsilon$ es el error. Al juntar las ecuaciones, la diferencia salarial media puede ser explicada por la diferencia media de los predictores de cada grupo ocupando la predicción lineal.

$$
\mathrm{D}=\mathrm{E}\left(\mathrm{Y}_{\mathrm{A}}\right)-\mathrm{E}\left(\mathrm{Y}_{\mathrm{B}}\right)=\mathrm{E}\left(\mathrm{X}_{\mathrm{A}}\right)^{\prime} \beta_{\mathrm{A}}-\mathrm{E}\left(\mathrm{X}_{\mathrm{B}}\right)^{\prime} \beta_{\mathrm{B}}
$$

y se considera que

$$
\mathrm{E}\left(\mathrm{Y}_{1}\right)=\mathrm{E}\left(\mathrm{X}_{l}^{\prime} \beta_{l}+\varepsilon_{l}\right)=\mathrm{E}\left(\mathrm{X}_{l}^{\prime} \beta_{l}\right)+\mathrm{E}\left(\varepsilon_{l}\right)=\beta_{l} \mathrm{E}\left(\mathrm{X}_{l}^{\prime}\right)
$$

Suponiendo que $\mathrm{E}\left(\beta_{l}\right)=\beta_{l}$ y $\mathrm{E}\left(\varepsilon_{l}\right)=0$, con lo expresado la doble descomposición es la que se utilizará, en la que se presume un vector de coeficientes no discriminatorios como $\beta^{*}$, por lo que se reorganiza a $\mathrm{D}$ y se expresa.

$$
\mathrm{D}=\left\{\mathrm{E}\left(\mathrm{X}_{\mathrm{A}}\right)+\mathrm{E}\left(\mathrm{X}_{\mathrm{B}}\right)\right\}^{\prime} \beta^{*}+\mathrm{E}\left(\mathrm{X}_{\mathrm{A}}\right)^{\prime}\left(\beta_{\mathrm{A}}-\beta^{*}\right)+\mathrm{E}\left(\mathrm{X}_{\mathrm{B}}\right)^{\prime}\left(\beta^{*}-\beta_{\mathrm{B}}\right)=\mathrm{Q}+\mathrm{U}
$$

Siendo $\mathrm{Q}=\left\{\mathrm{E}\left(\mathrm{X}_{\mathrm{A}}\right)+\mathrm{E}\left(\mathrm{X}_{\mathrm{B}}\right)\right\}^{\prime} \beta^{*}$; el primer componente que explica la diferencia entre los predictores de cada grupo; y $U=E\left(X_{A}\right)^{\prime}\left(\beta_{A}-\beta^{*}\right)+E\left(X_{B}\right)^{\prime}\left(\beta^{*}-\beta_{B}\right)$ el segundo componente la parte que no es explicada por los componentes. En la que recaen efectos de diferencias salariales de predictores que no se consideraron en la predicción lineal.

Asumiendo la estimación muestral, se considera que la discriminación se encuentra dirigida a un solo grupo de los dos estudiados, puntualmente se supone la discriminación negativa hacia mujeres y no existe discriminación positiva hacia hombres, por lo que se utiliza $\hat{\beta}_{\mathrm{A}}$ como estimado de $\beta^{*}$; quedando la ecuación de la siguiente manera:

$$
\widehat{\mathrm{D}}=\left(\bar{X}_{A}-\bar{X}_{B}\right)^{\prime} \hat{\beta}_{\mathrm{A}}+\hat{X}_{\mathrm{B}}^{\prime}\left(\hat{\beta}_{\mathrm{A}}-\hat{\beta}_{\mathrm{B}}\right)
$$

\section{Resultados y discusión}

Considerando la situación demográfica del Ecuador, en el periodo de estudio durante los años 2010 al 2019, de acuerdo al sexo, estaban dividido parcialmente en un 50\% tanto para hombres como para mujeres este porcentaje no tuvo varianza a lo largo del estudio de los años ya indicados. Por otra parte, la población en edad de trabajar (PET) estuvo conformada por un 52\% siendo este en número más alto y ocupándolo las mujeres, a diferencia de los hombres que contaron con un $48 \%$ INEC (2019). Analizando lo mencionado anteriormente la correspondencia ya no es la misma cuando nos referimos a la población económicamente activa, en la figura 1 se visualizó que los hombres cuentan con un 60,57\% y las mujeres con un 39,43\%. Siendo la participación activa liderada por la población de los hombres en la economía ecuatoriana. 


\section{Tabla 1}

Participación hombres y mujeres (\%) de población económicamente activa (PEA) Ecuador periodo 2010-2019

\begin{tabular}{lrrrrrrrrrr}
\hline & $\mathbf{2 0 1 0}$ & $\mathbf{2 0 1 1}$ & $\mathbf{2 0 1 2}$ & $\mathbf{2 0 1 3}$ & $\mathbf{2 0 1 4}$ & $\mathbf{2 0 1 5}$ & $\mathbf{2 0 1 6}$ & $\mathbf{2 0 1 7}$ & $\mathbf{2 0 1 8}$ & $\mathbf{2 0 1 9}$ \\
\hline Hombres & $60,57 \%$ & $60,41 \%$ & $60,26 \%$ & $60,65 \%$ & $59,77 \%$ & $59,30 \%$ & $58,18 \%$ & $57,71 \%$ & $58,41 \%$ & $58,18 \%$ \\
\hline Mujeres & $39,43 \%$ & $39,59 \%$ & $39,74 \%$ & $39,35 \%$ & $40,23 \%$ & $40,70 \%$ & $41,82 \%$ & $42,29 \%$ & $41,59 \%$ & $41,82 \%$
\end{tabular}

Fuente: Elaboración propia con datos de encuesta nacional de estadísticas y censo - INEC; encuesta nacional de empleo, desempleo y subempleo - ENEMDU.

\section{Figura 1}

Participación femenina y masculina (\%) de población económicamente activa (PEA) Ecuador periodo 2010-2019

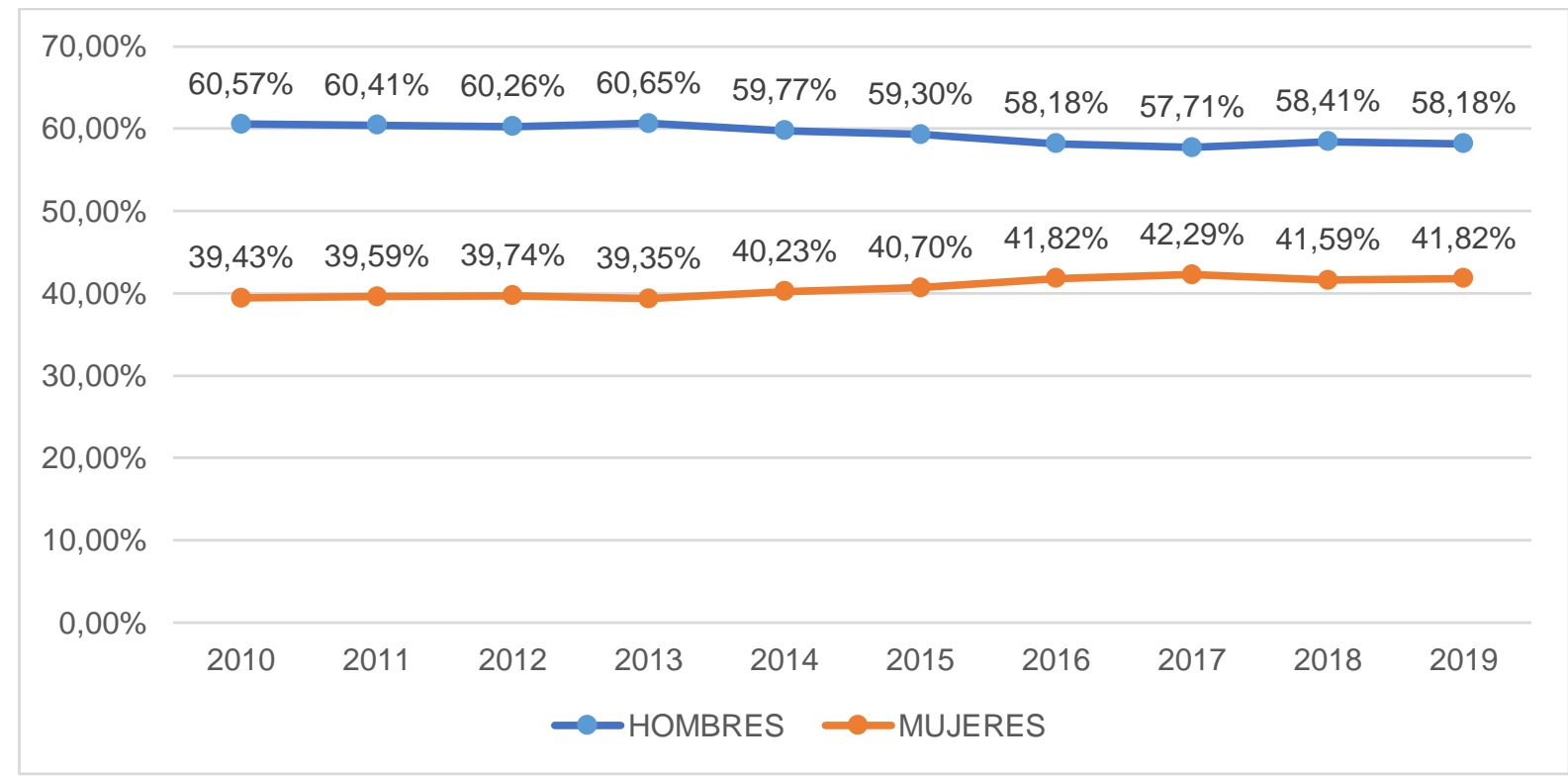

Fuente: Elaboración propia con datos de encuesta nacional de estadísticas y censo - INEC; encuesta nacional de empleo, desempleo y subempleo - ENEMDU.

En tabla 2 nos muestra los tabulados del mercado laboral respecto a la población económicamente activa (PEA) y población en edad de trabajar con la que se determinó la tasa de actividad que es el cociente entre la población activa y la población en edad de trabajar o mayor de 16 años. 
Tabla 2

Tabulados del mercado laboral ecuatoriano periodo 2010-2019

\begin{tabular}{ccccc}
\hline ANOS & GENERO & PEA & PET & TASA DE ACTIVIDAD \\
\hline \multirow{2}{*}{2010} & Hombres & 3898390,4 & 5003062,0 & $77,92 \%$ \\
& Mujeres & 2537866,4 & 5288438,4 & $47,99 \%$ \\
2011 & Hombres & 3976254,0 & 5081307,1 & $78,25 \%$ \\
\multirow{2}{*}{2012} & Mujeres & 2605367,2 & 5451696,3 & $47,79 \%$ \\
\multirow{2}{*}{2013} & Hombres & 4038252,9 & 5251766,9 & $76,89 \%$ \\
& Mujeres & 2662761,2 & 5612380,2 & $47,44 \%$ \\
2014 & Hombres & 4217043,3 & 5461984,9 & $77,21 \%$ \\
& Mujeres & 2735942,3 & 5738385,7 & $47,68 \%$ \\
\multirow{2}{*}{2015} & Hombres & 4300115,6 & 5396146,7 & $79,69 \%$ \\
& Mujeres & 2894405,3 & 5763108,0 & $50,22 \%$ \\
\multirow{2}{*}{2016} & Hombres & 4446869,3 & 5534518,7 & $80,35 \%$ \\
& Mujeres & 3051658,7 & 5864757,6 & $52,03 \%$ \\
2018 & Hombres & 4581100,4 & 5685863,6 & $80,57 \%$ \\
& Mujeres & 3292920,4 & 6010267,5 & $54,79 \%$ \\
2019 & Hombres & 4666457,4 & 5787404,5 & $80,63 \%$ \\
& Mujeres & 3419590,7 & 6150523,6 & $55,60 \%$ \\
& Hombres & 4688579,9 & 5985669,0 & $78,33 \%$ \\
& Mujeres & 3338549,9 & 6253354,0 & $53,39 \%$ \\
& Hombres & 4711946,7 & 6066275,0 & $77,67 \%$ \\
\hline
\end{tabular}

Fuente: Elaboración propia con datos de encuesta nacional de estadísticas y censo - INEC; encuesta nacional de empleo, desempleo y subempleo - ENEMDU.

Observando los resultados de tasa de actividad de hombres y mujeres en la figura 2 , muestra que del total de hombres y mujeres que se encuentran en actividad trabajar en promedio en los años de estudio para los hombres es de 78,75\% y del 51,04\% de las mujeres que se encuentran activando la economía. Existiendo una diferencia de participación porcentual entre hombres y mujeres de $27,71 \%$.

De acuerdo al INEC de todas las personas que se encuentran económicamente activas de cualquier sexo, existe un segmento que se encuentran con empleo con o sin remuneración, y que se dedicaban a alguna actividad para producir bienes o prestar servicios, con empleo «trabajando», por lo menos una hora, y con empleo pero «sin trabajar» por ausencia temporal del puesto de trabajo o por otra causas como trabajo en turnos, horarios flexibles y licencias compensatorias por horas extraordinarias, es así que una parte de la PEA que concuerda con estas características es denomina población empleada. 


\section{Figura 2}

Tasa de actividad (\%) de hombres y mujeres del Ecuador periodo 2010-2019

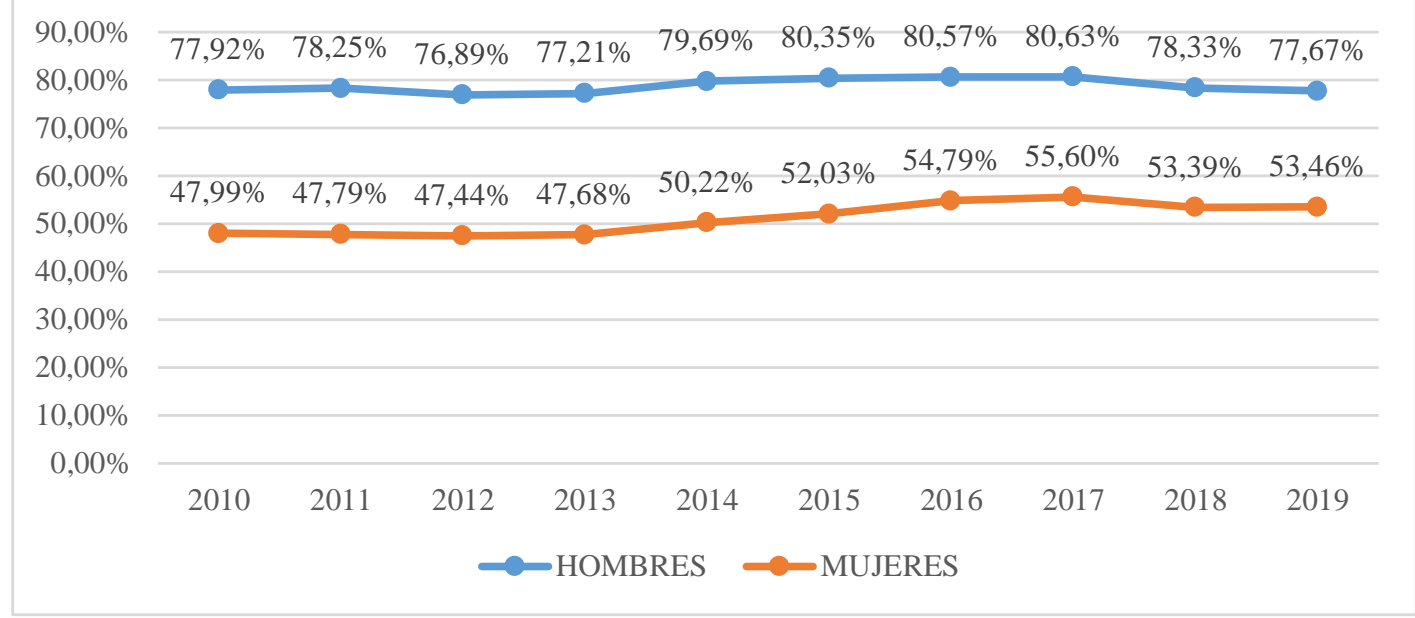

Fuente: Elaboración propia con datos de encuesta nacional de estadísticas y censo - INEC; encuesta nacional de empleo, desempleo y subempleo - ENEMDU.

De igual forma que en la composición de la PEA, la composición de la población empleada figura 3, se encuentra conformada en promedio con un 59,81\% de hombres y un 40,19\% de mujeres. Con lleva a que existe un panorama en el que se expone una diferencia entre hombres y mujeres orientando al desempleo abierto. Las mujeres se encuentran más propensas a estar desempleadas por inconvenientes o dificultades en permanecer o mantenerse en el trabajo, de acuerdo Aquilini y Costa (2003) es el sexo femenino que tiene mayor número de desempleados e inactivas, ya sea por la inclusión en la actividad laboral o por la responsabilidad de la vida familiar con el cuidado de los hijos.

\section{Figura 3}

Composición de la población ocupada en Ecuador periodo 2010-2019

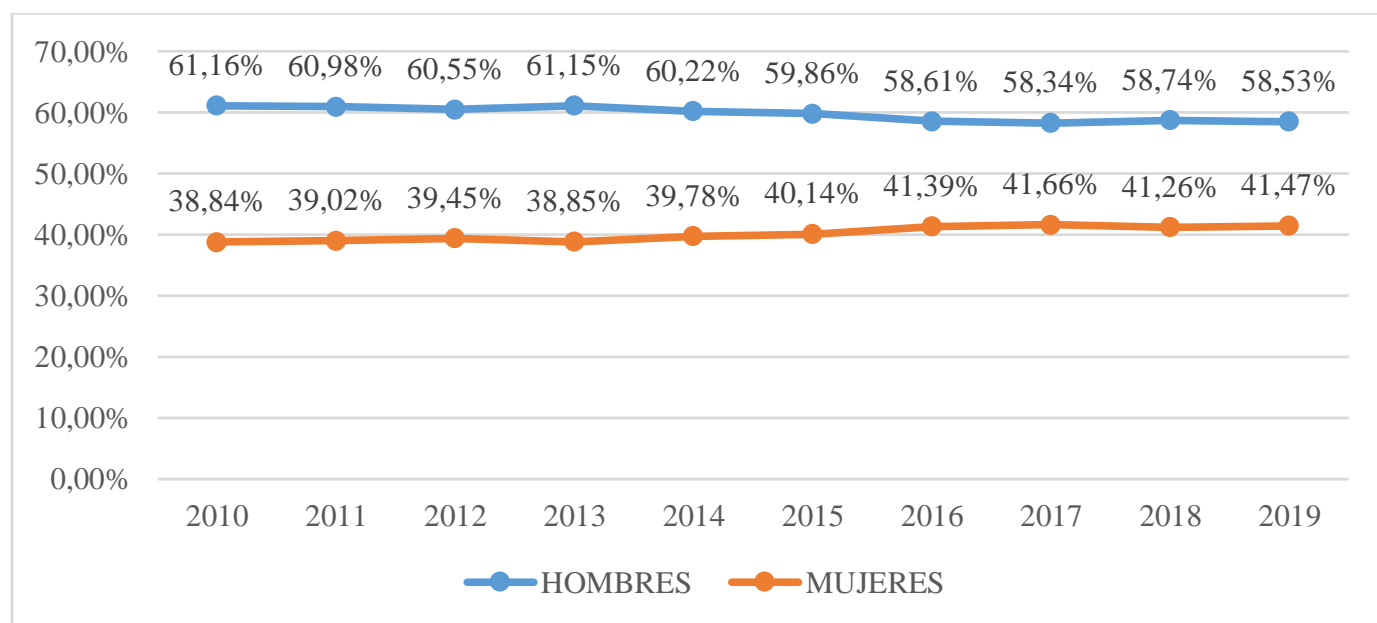

Fuente: Elaboración propia con datos de encuesta nacional de estadísticas y censo - INEC; encuesta nacional de empleo, desempleo y subempleo - ENEMDU. 
Se puede mencionar de acuerdo a la figura 4, el desempleo de hombres en promedio en el Ecuador alcanza el 49,09\% y el de las mujeres llegan al 50,91\% en promedio, el desempleo tanto para hombres como para mujeres se ha mantenido estable durante estos dos últimos años con valores oscilantes entre 49,62\% y 50,38\%. En los años de estudio la cantidad de hombres laborando es mayor que el de mujeres, identificando una marcada diferencia en lo laboral. Con esto, la suma del total de ingresos de los hombres es mayor que el de las mujeres, y para próximos cálculos se utilizará la metodología Oaxaca-Blinder en el que se tendrá el ingreso medio como un apropiado indicador analítico.

\section{Figura 4}

Desempleo (\%) de hombres y mujeres en Ecuador periodo 2010-2019

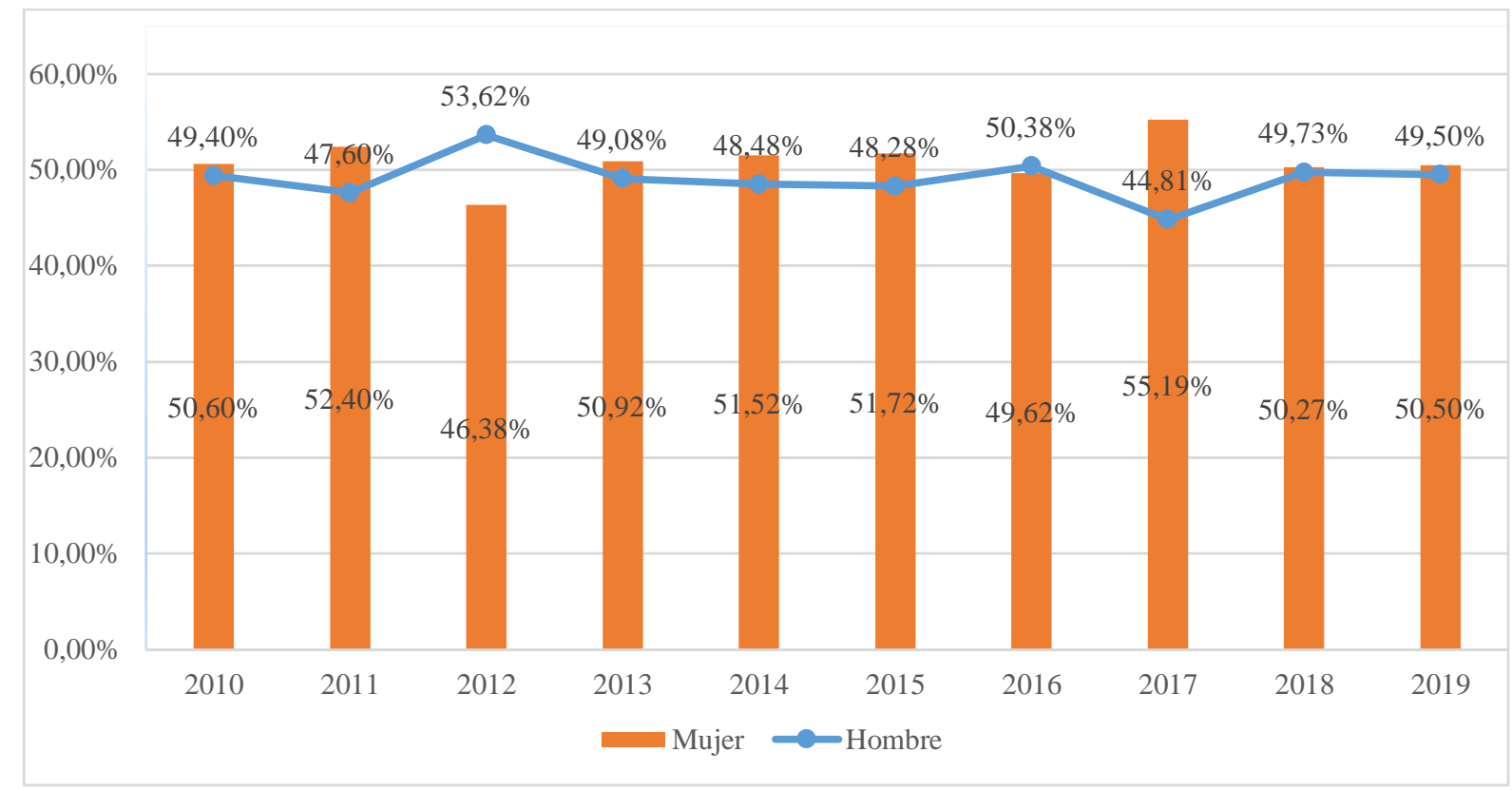

Fuente: Elaboración propia con datos de encuesta nacional de estadísticas y censo - INEC; encuesta nacional de empleo, desempleo y subempleo - ENEMDU.

\section{Diferencia salarial, ingresos de hombres y mujeres}

La distribución de los hombres y mujeres en el área laboral, se la considera una variable muy importante al momento de determinar la brecha salarial de género. Como se puede observar en la figura de los ingresos medios, se visualiza que los hombres se encuentran por mayoría trabajando en el mercado, obteniendo de esa manera mayores ingresos, debido al cargo o puesto que ocupen. 


\section{Tabla 3}

Promedio mensual del ingreso laboral, expresados en valores reales año base 2007

\begin{tabular}{lcrrrrrrrrr}
\hline & $\mathbf{2 0 1 0}$ & $\mathbf{2 0 1 1}$ & $\mathbf{2 0 1 2}$ & $\mathbf{2 0 1 3}$ & $\mathbf{2 0 1 4}$ & $\mathbf{2 0 1 5}$ & $\mathbf{2 0 1 6}$ & $\mathbf{2 0 1 7}$ & $\mathbf{2 0 1 8}$ & $\mathbf{2 0 1 9}$ \\
\hline Hombres & 329,64 & 331,67 & 341,88 & 381,65 & 384,81 & 388,17 & 367,70 & 369,34 & 357,53 & 345,48 \\
Mujeres & 268,31 & 262,77 & 283,15 & 301,26 & 296,29 & 300,75 & 287,18 & 295,44 & 293,56 & 292,71 \\
\hline Total & 307,70 & 306,82 & 320,40 & 352,63 & 351,96 & 355,58 & 337,12 & 341,25 & 333,53 & 325,77 \\
\hline
\end{tabular}

Fuente: Elaboración propia con datos de encuesta nacional de estadísticas y censo - INEC; encuesta nacional de empleo, desempleo y subempleo - ENEMDU.

\section{Figura 5}

Promedio mensual del ingreso laboral de hombres y mujeres en Ecuador. Expresados en valores reales diciembre 2007

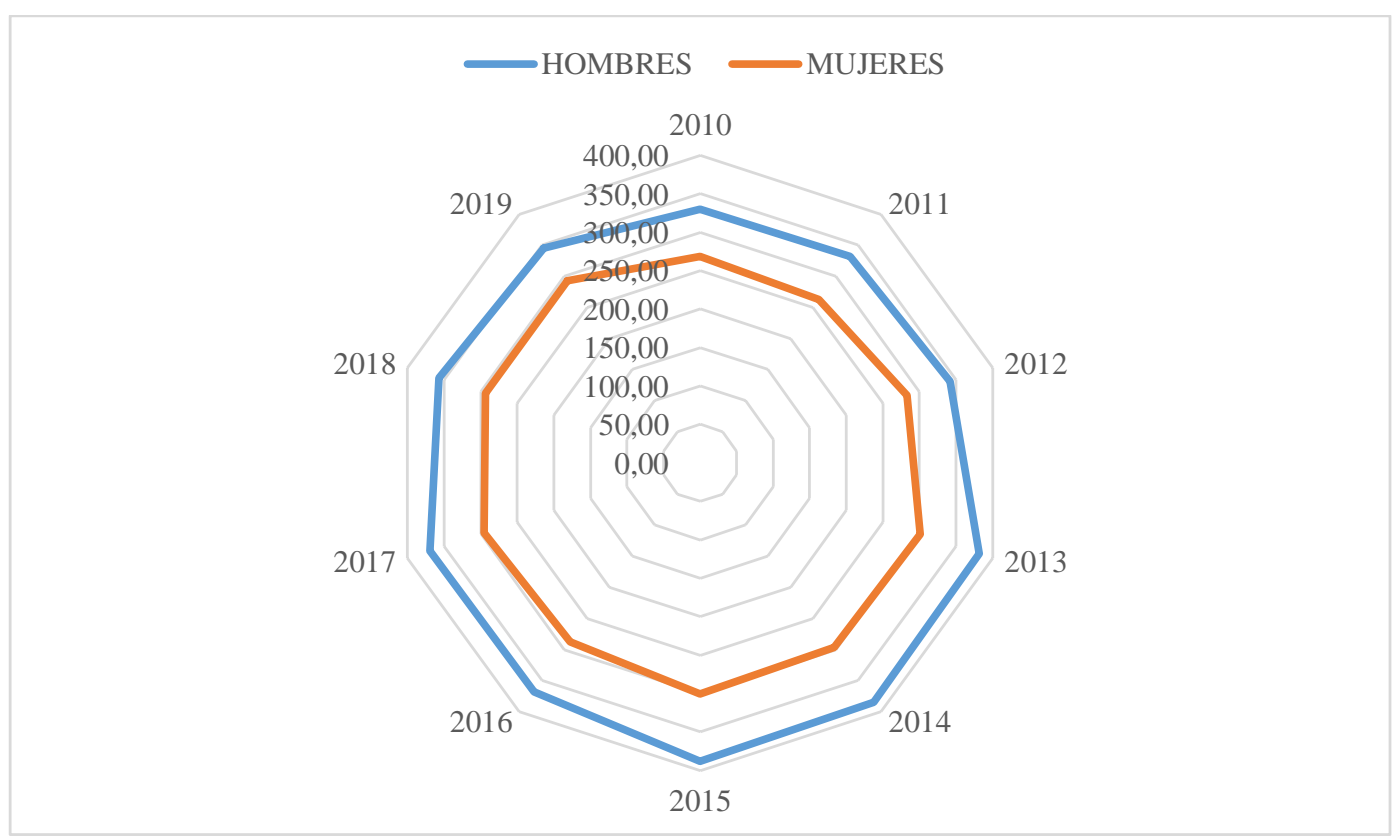

Fuente: Elaboración propia con datos de encuesta nacional de estadísticas y censo - INEC; encuesta nacional de empleo, desempleo y subempleo - ENEMDU.

Como podemos notar, en la figura 5, los hombres han tenido un mayor ingreso en el año 2015 cuyo monto esta alrededor de 400 dólares, a diferencia de las mujeres que en ese mismo periodo se encontraba con un salario de 300 dólares. Analizando otro punto de vista, se puede recalcar, que los hombres perciben un mayor salario por los trabajos que realizan, ya sean estos de nivel tecnológico o por responsabilidad de los cargos. 


\section{Tabla 4}

Resultados de la descomposición de la economía de ecuatoriana del año 2015

\begin{tabular}{lccccccc}
\hline & & \multicolumn{3}{c}{ Hombres } & \multicolumn{3}{c}{ Mujeres } \\
\hline Salario & $\mathbf{N}$ & $\mathbf{\%}$ & Media & SD & \% & Media & SD \\
& 2.160 .032 & 62,01 & 839 & 1,011 & 37,99 & 845 & 758 \\
Solteros & 1.031 .109 & 63,03 & 647 & 592 & 36,97 & 739 & 593 \\
Casados & 964.080 & 63,59 & 1.020 & 1283 & 36,41 & 924 & 842 \\
Divorciados & 139.651 & 47,49 & 1.043 & 1084 & 52,51 & 1.006 & 978 \\
Viudos & 19.863 & 33,98 & 867 & 977 & 66,02 & 867 & 837 \\
Unión Libre & 5.329 & 64,68 & 883 & 879 & 35,32 & 829 & 687 \\
& & & & & & & \\
Título & & & & & & & \\
Profesional & & & & & & & \\
Técnico & 73.402 & 47,53 & 1.004 & 848 & 52,47 & 801 & 550 \\
Tercer nivel & 442.231 & 40,69 & 1.485 & 1262 & 59,31 & 1.105 & 786 \\
Cuarto nivel & 77.046 & 47,94 & 2.364 & 1.948 & 52,06 & 1.767 & 1.283 \\
Sin título & 1.567 .353 & 69,40 & 675 & 818 & 30,60 & 628 & 573 \\
& & & & & & & \\
Tipo Título & & & & & & & \\
Nacional & 576.789 & 42,04 & 1.512 & 1.354 & 57,96 & 1.132 & 851 \\
Extranjero & 15.890 & 58,41 & 2.472 & 1.928 & 41,59 & 1.998 & 1.408 \\
Edad & 2.160 .032 & 62,01 & 37 & 12 & 37,99 & 37 & 11 \\
\hline
\end{tabular}

\section{Conclusiones}

El propósito de este estudio fue analizar la relación entre las diferencias salariales entre hombres y mujeres y la segregación ocupacional de utilizando datos detallados sobre ocupaciones. Se estimaron las brechas salariales de género por separado de la población económicamente activa 2010 - 2019 y contrastaron las diferencias en los salarios y las características de los empleados. Luego se analizó en porcentajes la tasa de actividad de hombres y mujeres del Ecuador periodo 2010-2019, mediante la relación de las personas que se encuentran trabajando y sin trabajar en el país de acuerdo a datos oficiales del INEC. Los valores indican que los salarios de hombres y mujeres son los más altos en ocupaciones donde se requiere el nivel más alto de educación e inversiones en el trabajo, más, sin embargo, la relación entre el nivel de salarios promedio y la segregación ocupacional parece ser bastante débil.

Los resultados de la descomposición Oaxaca-Blinder muestran que, una parte muy pequeña de las diferencias salariales entre hombres y mujeres puede explicarse por diferencias en las características personales y empresariales. Al comparar el tamaño de la parte inexplicada de la brecha salarial de género identificada en la literatura con el efecto de discriminación salarial y 
la tasa de masculinización, los autores encontraron relaciones no lineales muy débiles entre estos dos indicadores. La parte inexplicable de la brecha salarial de género es la más pequeña en ocupaciones fuertemente dominadas por mujeres y en ocupaciones con una relación hombremujer casi equilibrada. Los efectos de discriminación parecen ser relativamente altos en ocupaciones dominadas por hombres y mujeres, con algunos indicios de que la relación analizada es ligeramente positiva.

Existen diferencias inexplicables de acuerdo a los resultados obtenido en los salarios entre hombres y mujeres, lo que pone en tela de juicio el fenómeno de discriminación salarial en el mercado laboral ecuatoriano. Las diferencias salariales parecen ser, en gran medida, el resultado de otros factores difíciles de incluir en el modelo, como las diferencias en las condiciones psicofísicas de hombres y mujeres, antecedentes culturales, tradiciones o hábitos. Las que podrían explicar, por ejemplo, diferencias relativamente altas en los salarios de hombres y mujeres donde se requiere fuerza física adicional como empleados calificados y operadores de máquinas.

Las diferencias inexplicables relativamente altas en los salarios de hombres y mujeres en algunas ocupaciones directivas, departamentales, ejecutivas y profesionales también podrían justificarse por la naturaleza de estos trabajos que consume mucho tiempo y el hecho de que más mujeres que hombres tienen que conciliar el trabajo y la vida familiar. No tener en cuenta estos factores puede dar lugar a una interpretación excesiva de la parte no explicada como una medida de discriminación de género.

\section{Referencias Bibliográficas}

Aguilar, A. C., \& Vera., M. A. (2016). Diferencias Salariales por genero en el departamento de Santander, Colombia. Apuntes del CENES, 267-301. Obtenido de https://www.redalyc.org/articulo.oa?id=479555352010

Albuja-Echeverría, W., \& Enríquez-Rodríguez, M. J. (2018). Análisis de la discriminación laboral hacia las mujeres en Ecuador 2007-2016. Convergencia, 25(78), 13-41.

Alcañiz, M., \& Monteiro, R. (2016). She-austerity. Precariedad y desigualdad laboral de las mujeres en el sur de Europa. CONVERGENCIA, 23(72), 39-67.

Angulo, G., Quejada, R., \& Yánez, M. (2012). Educación, mercado de trabajo y satisfacción laboral: el problema de las teorías del capital humano y señalización de mercado. Revista de la educación superior, 41(163), 51-66.

Anker, R. (1997). Women, gender and work, What is equality and how do we get there ? En M. Fetherolf Loutfi, Theories of occupational segregation by sex: An overview (Vol. 136).

Aquilini, G., \& Costa, P. (2003). O sobre-desemprego e a inatividade das mulheres na metrópole paulista. Estudos Avançados, 17(49), 17-34.

Banerjee, B. (2014). Occupational segregation and gender differentials in earnings in Macedonia. IZA Journal of European Labor Studies, 3(4), 1-27. 
Barón, J., \& Cobb-Clark, D. (2010). Occupational segregation and the gender wage gap in privateand public-sector employment: a distributional analysis. The Economic Record, 86(273), 227-246.

Bergmann, B. (1986). The Economic Emergence of Women. Basic Books Inc. New York, NY.

Blau, F., \& Kahn, L. (2003). Understanding international differences in the gender pay gap. Journal of Labour Economics, 21, 106-144.

Blinder, A. (1973). Wage discrimination: reduced form and structural estimates. Journal of Human Resources, 8(4), 436-455.

Brynin, M., \& Perales, F. (2016). Gender Wage Inequality: The De-gendering of the Occupational Structure. European Sociological Review, 32(1), 162-174.

Bustos, C. (2008). Las diferencias salariales en PROULA Medicamentos. ACTUALIDAD Contable Faces, 11-27. Obtenido de https://www.redalyc.org/pdf/257/25701603.pdf

Caicedo, M. (2015). La desigualdad salarial entre inmigrantes latinoamericanos y nativos en Estados Unidos (1980-2010). NORTEAMÉRICA. Revista Académica del CISAN - UNAM, 133-164. Obtenido de https://www.redalyc.org/pdf/1937/193742701005.pdf

Constitucion de la Republica del Ecuador. (2008, última modificación 2015). Registro Oficial 449. Quito, Ecuador.

England, P., Herbert, M. S., Kilbourne, B. S., \& L, M. M. (1994). The gendered valuation of occupations and skill earnings in 1980 census occupations. Social Forces, 73(1), 65-100.

Evertsson, M., England, P., Mooi-Reci, I., Hermsen, J., de Bruijn, J., \& Cotter, D. (2009). Is gender inequality greater at lower or higher educational levels? Common patterns in the Netherlands, Sweden, and the United States. Social Politics, 16(2), 210-241.

García, J., \& Cortez, P. (2011). Análisis de la participación laboral de la mujer en el mercado ecuatoriano. Analítika, 4(2), 27-53.

Garrido, C. (2007). La educación desde la teoría del capital humano y el otro. Educere, 11(36), 73-80.

Goraus, K., Tyrowicz, J., \& Van der Velde, L. (2017). Which gender wage gap estimates to trust? A comparative analysis. Review of Income and Wealth, 63(1), 118-146.

Hakim, C. (1998). Social change and innovation in the labour market: evidence from the census SARs on occupational segregation and labour mobility, part-time work and students' jobs, homework and self-employment. OUP Catalogue.

INEC. (2019). Instituto Nacional de Estadística y Censo. Quito, Ecuador.

Juárez, C., \& Aboites, G. (2008). Segregacion Ocupacional en la Zona Metropolitana de Saltillo. Papeles de población, 14(57), 209-225. Obtenido de http://www.scielo.org.mx/pdf/pp/v14n57/v14n57a10.pdf

Jumbo, D. E., \& Granda, D. M. (2014). Diferencias Salariales regionales ligadas a la educacion: aplicacion a la region sur del Ecuador, 2010. REVISTA ANALISIS ECONOMICO, 185-202. Obtenido de https://www.redalyc.org/pdf/413/41331851010.pdf

Jurajda, S. (2003). Gender wage gap and segregation in enterprises and the public sector in late transition countries. Journal of Comparative Economics, 31(2), 199-222.

Kunze, A. (2008). Gender wage gap studies: consistency and decomposition. Empiral Economic, $35(63-76)$. 
Lavassani, K. M., \& Mohavedi, B. (2014). Developments in theories and measures of workfamily relationships: From conflict to balance. Contemporary Research on Organization, 2(2), 619.

Ley orgánica integral para prevenir y erradicar la violencia contra las mujeres, R. O. (2018). Registro Oficial 175. Quito, Ecuador.

López, J., \& Sarmiento, G. (2019). Determinantes de la brecha salarial en Ecuador: análisis bajo un modelo minceriano relacionado con variables agregadas dummy. Espirales revista multidisciplinaria de investigación científica, 3(27), 78-89.

Milan Vásquez de la Torre, M., Santos, M., \& Perez, L. (2015). Análisis del mercado laboral femenino en España: evolución y factores socioeconómicos determinantes del empleo. Papeles de Poblacion, 21(84), 197- 225.

Mincer, J., \& Polachek, S. (1974). Family investments in human capital: earnings of women. Journal of Political Economy, 82(2), 76-108.

Murphy, E., \& Oesch, D. (2015). The feminization of occupations and change in wages: a panel analysis of Britain, Germany and Switzerland (Vol. 731). Berlin.

Novillo, E., Sarmiento, C., Ollague, J., \& Ramón, D. (2017). Análisis de actitud emprendedora en estudiantes universitarios: una perspectiva de género. INNOVA, Research Journal, 2(8.1), 56-63.

Oaxaca, R. (1973). Male-female wage differentials in urban labor markets. International Economic Review, 14(3), 693-709.

Ochsenfeld, F. (2014). Why do women's fields of study pay less? A test of devaluation, human capital, and gender role theory . European Sociological Review , 30, 536-548.

Ospina, E. (2015). Capital humano: una visión desde la teoría crítica. Cadernos EBAPE,BR, 13(2), 315-331.

Oyinlade, A. O., \& Losen, A. (2014). Extraneous Effects of Race, Gender, and Race-Gender Homo- and Heterophily Conditions on Data Quality. SAGE Open, 4(1), 1-13.

Simón, H., Ramos, R., \& Sanromá, E. (2008). Evolución de las diferencias salariales por razón de sexo. Revista de Economia Aplicada, XVI(48), 37-68.

Sławińska, K. (2020). Public-private sector wage gap in a group of European countries: an empirical perspective. Empiral Economic.

Tam, T. (1997). Sex segregation and occupational gender inequality in the United States: devaluation or specialized training? . American Journal of Sociology, 102, 1652 - 1692.

Uriguen, P., Vega, F., \& Luna-Romero, A. (2020). El uso de las TIC en el aprendizaje en la Universidad caso UTMACH. INNOVA, Research Journal, 5(1), 45-60.

Weichselbaumer, D., \& Winter-Ebner, R. (2005). A meta-analysis of the international gender wage gap . Journal of Economic Surveys, 19, 479-511.

Zuñiga, P. C. (2006). Mujeres Ecuatorianas: Panorama de situaciones y perspectivas finales del siglo XX y principios del siglo XXI. Araucaria. Revista Iberoamericana de Filosofia, Politica $\quad y \quad$ Humanidades, 190-211. Obtenido de https://www.redalyc.org/pdf/282/28281610.pdf 